\title{
RESULTS FROM COMMISSIONING THE AGS BOOSTER ORBIT SYSTEM*
}

\author{
E. Bleser \\ AGS Department, Brookhaven National Laboratory \\ Upton, NY 11973
}

\section{INTRODUCTION}

This note reports results from the commissioning of three systems in the AGS Booster [1]: the beam position monitor system, which works to a relative accuracy of 0.36 millimeters; the uncorrected Booster orbit, which has quite large excursions; and the passive eddy current correction system, which eliminates all but a few percent of the eddy current dipole effect.

\section{RESULTS FROM \\ THE BEAM POSITION MONITOR SYSTEM}

Figure 1 shows an uncorrected orbit in the Booster as measured by the beam position monitor (BPM) system [2]. It has excursions of $\pm 15 \mathrm{~mm}$ where $\pm 5 \mathrm{~mm}$ would have been excessive. This orbit is fully correctable by the steering magnet system so it is not deleterious to machine operation but it does raise several questions as to the validity of the BPM system, the quality of the maynets, and the accuracy of the survev. In a new machine all problems are possible.

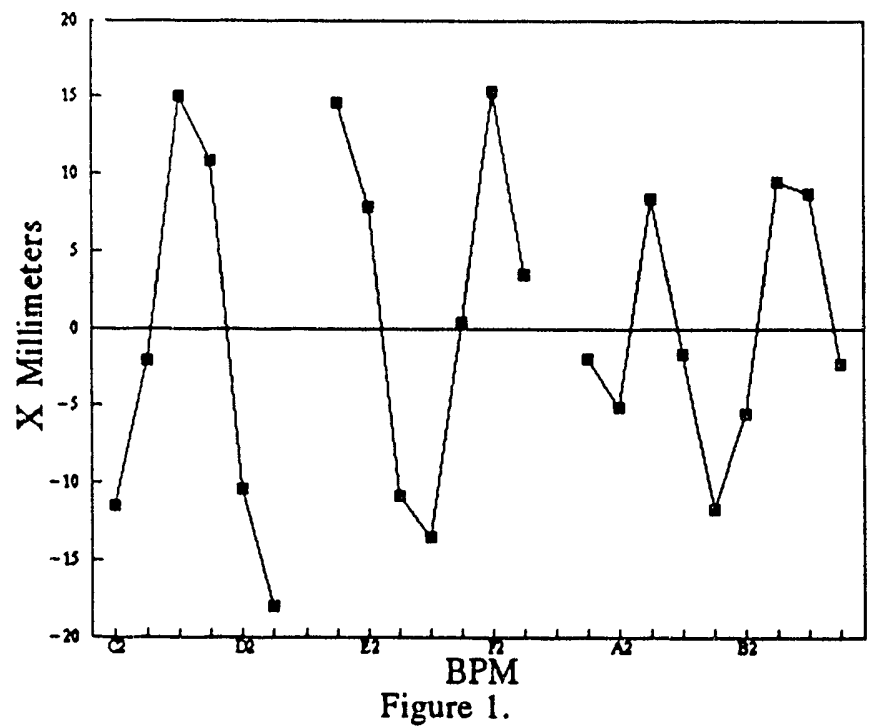

A measurement of the uncorrected orbit of the proton beam in the AGS Booster. BPM's D6 and F6 do not exist because of the requirements of the dump and extraction systems.

*Work performed under the auspices of the U.S. Dept. of Energy.
In order to check out the BPM system the four extraction bump magnets were each powered individually and a difference orbit was found by subtracting the unperturbed orbit from the bumped orbit. A typical result is shown in Figure 2.

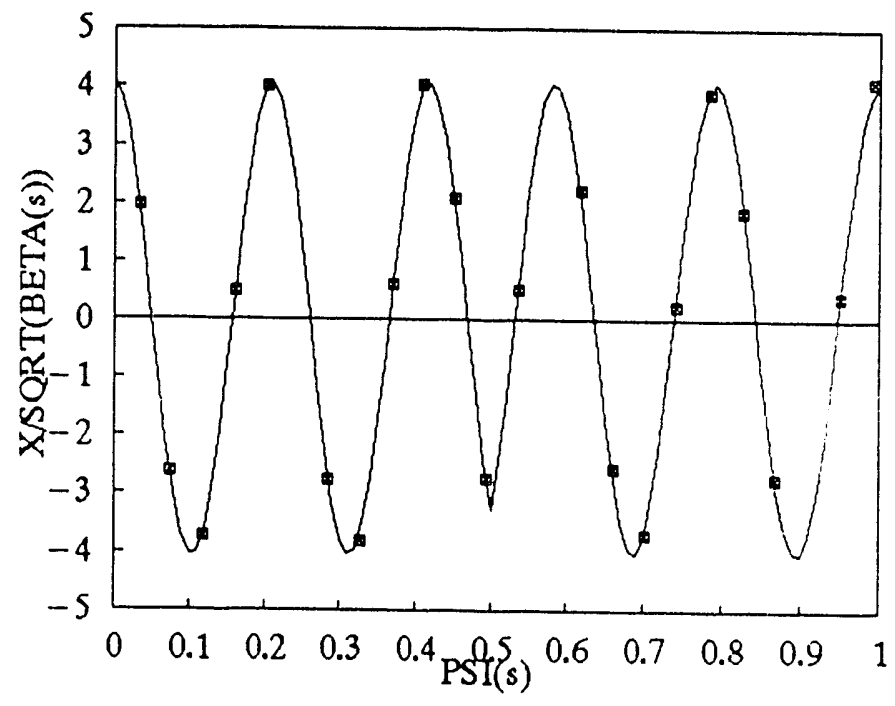

Figure 2.

A difference orbit showing the effect of a bump magnet. The $Y$-axis is the difference, $X$, in millimeters divided by the square root of the local Twiss beta function in meters. The $X$ axis is $\psi$, the phase advance around the machine divided by $2 \pi$ $Q$, where $Q$ is the tune. The bump magnet is located at $\psi=$ 0.5. The formula: [3] $X(s)=K \cos (2 \pi Q * \psi)$ is fitted over the range $-0.5<\psi<+0.5$.

We can assume that the output of a BPM is given by:

$$
x=a+b * x
$$

where $a$ and $b$ are constants, preferably 0 and 1 , and $x$ is the actual position of the beam in the BPM. We can evaluate each BPM by powering each of the four magnets at two different currents, assuming the actual beam position, $x$, is that given by the curve fitted to all twenty-two points, and then compar- 
ing the measured position against that called for by the fit. Figure 3 shows a typical result. In sum the BPM's are linear over a range of $\pm 25 \mathrm{~mm}$, the average value of $b$ is 1.006 \pm .03 , and for difference orbits the BPM measures a change in position to an accuracy of $0.36 \mathrm{~mm}$. In addition this process calibrates the extraction bumps in situ to an accuracy of $1 \%$.

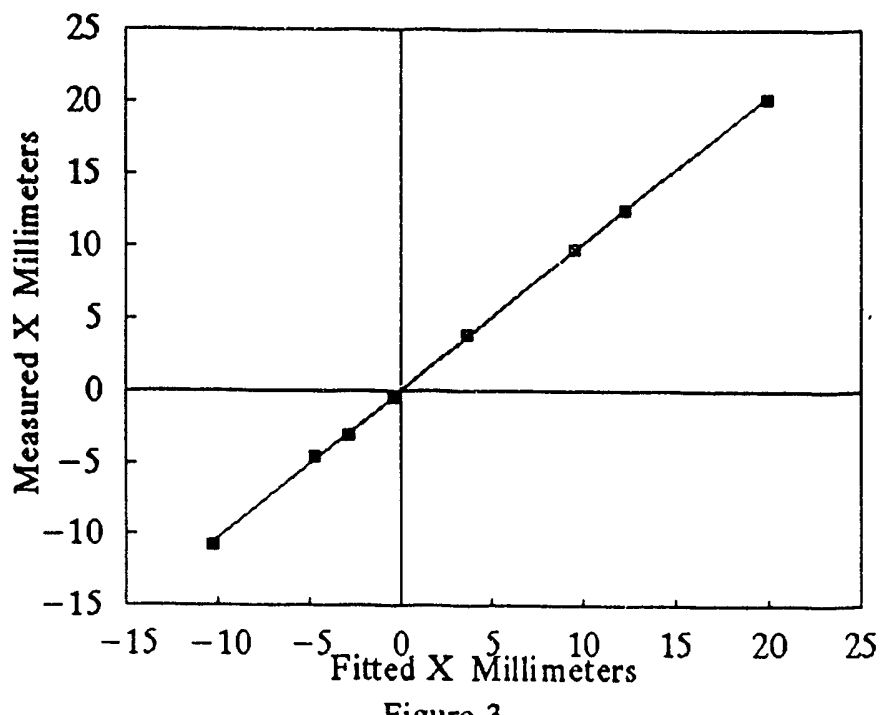

Figure 3.

A plot for one BPM of X measured for various bumps versus the beam position predicted by fitting the formula of Figure 2 to all 22 BPM's.

\section{EDDY CURRENT EFFECTS}

We can conclude that the BPM system is very good for difference orbits but we still do not have any information on the absolute orbit, shown in Figure 1, which can have many possible sources - field errors, survey errors, or offset terms, $a$, in the BPM's. Before pursuing this question we can evaluate the absolute orbit as a function of magnetic field strength, $B$, and as a function of rate of change of field, $\mathrm{dB} / \mathrm{dt}$.

The Booster is a rapid cycling machine, going from 600 to 5000 gauss in 60 milliseconds, and it has stainless steel vacuum chamber in the field. To compensate for the high eddy currents produced in the vacuum chamber, a passive correction system [4] has been installed which consists of field windings placed on the chamber, which windings are powered by windings around the magnet poles. The system is designed to balance the sextupole component induced by currents in the vacuum chambers. Thus there are four contribution to the dipole field: 1 . The main field of the magnets, by far the largest; 2 . The dipole field produced by the currents induced in the vacuum chamber, which retards the main field; 3 . The dipole field produced by the currents induced in the windings around the magnet poles, which also retard; the main field; 4. The dipole field produced by the windings placed on the vacuum chamber, which are connected so as to add to the main field. As long as these effects are uniform from magnet to magnet they are of no consequence and there should be no observable orbit dependence on $\mathrm{dB} / \mathrm{dt}$. However, comparing orbits at two different values of $\mathrm{dB} / \mathrm{dt}$ gives the result shown in Figure 4, a significant effect. The orbit can be nicely fit by assuming a dipole, proportional to $\mathrm{dB} / \mathrm{dt}$, located at the injection magnet, a special magnet with a special vacuum chamber. Subsequent, unrelated work discovered that the correction system for this special case was miswired. After fixing this case there still remained a small dependence on $\mathrm{dB} / \mathrm{dt}$, which we attribute to random errors in the eddy currents. In particular for the Booster this orbit corresponds to random bend errors of 0.01 milliradians or .2 Gauss. Table 1 summarizes the expected eddy current fields. We can conclude that the individual elements are good to $3 \%$ to produce this small an orbital effect. These effects are not significant for the Booster orbit but they do enable us to project that the eddy current: sextupoles are corrected to $3 \%$.

Table 1.

DIPOLE EDDY CURRENT FIELDS

\begin{tabular}{||c|c|}
\hline \multicolumn{1}{|c|}{ SOURCE } & $\begin{array}{c}\text { FIELD } \\
\text { GAUSS }\end{array}$ \\
\hline Vacuum Chambei $(7 \mathrm{G} / \mathrm{ms})$ & -20 \\
\hline Correction Winding & +5 \\
\hline Random Error from Orbit & 0.2 \\
\hline
\end{tabular}

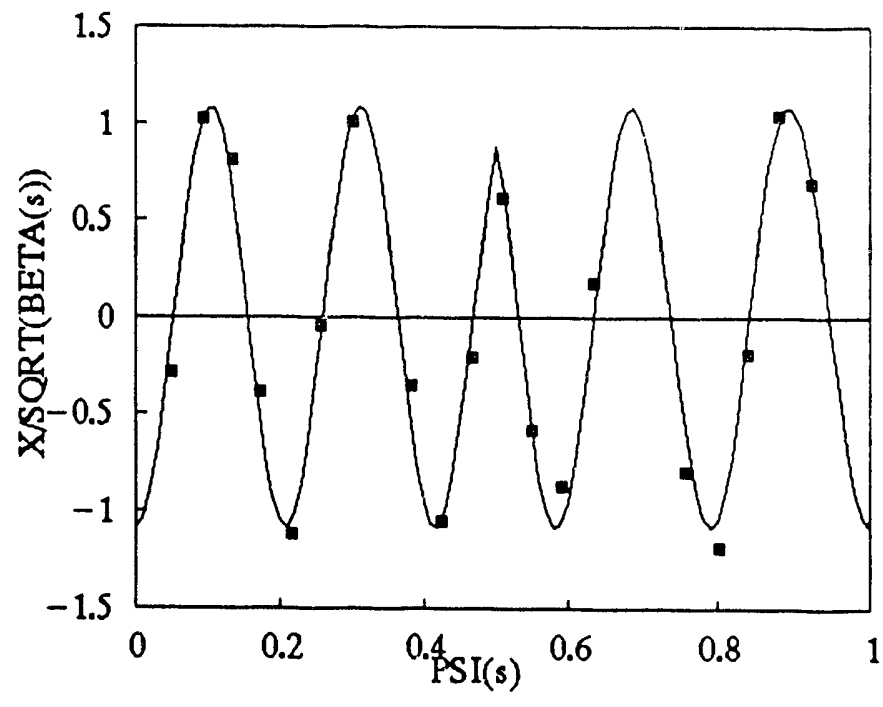

Figure 4

A difference orbit comparing uncorrected orbits as in Figure 1 taken at $\mathrm{dB} / \mathrm{dt}$ values of $5 \mathrm{G} / \mathrm{ms}$ and $70 \mathrm{G} / \mathrm{ms}$. The original orbits look quite similar and a significant difference becomes apparent only on subtraction. The curve is generated as in Figure 2 by assuming a bump at the injection magnet. 


\section{CONCLUSION}

Because several aspects of the orbit were easily explained, a fair effort was made to find a simple source for the orbit of Figure 1, but without success. In the Booster design manual, randoin survey errors for quadrupoles of 0.3 millimeters rms were assumed and 20 random distributions were calculated. The worst result is shown in figure 5, and it agrees remarkably well with our measured orbit. Apparently the simplest explanation for our observed orbit is that the random alignment errors have occurred in such a way that we have built a machine with an orbit in the fifth percentile. To maintain our professional pride we shall realign it in the next major shutdown, and hope that the gods of chance are more favorably disposed toward us.

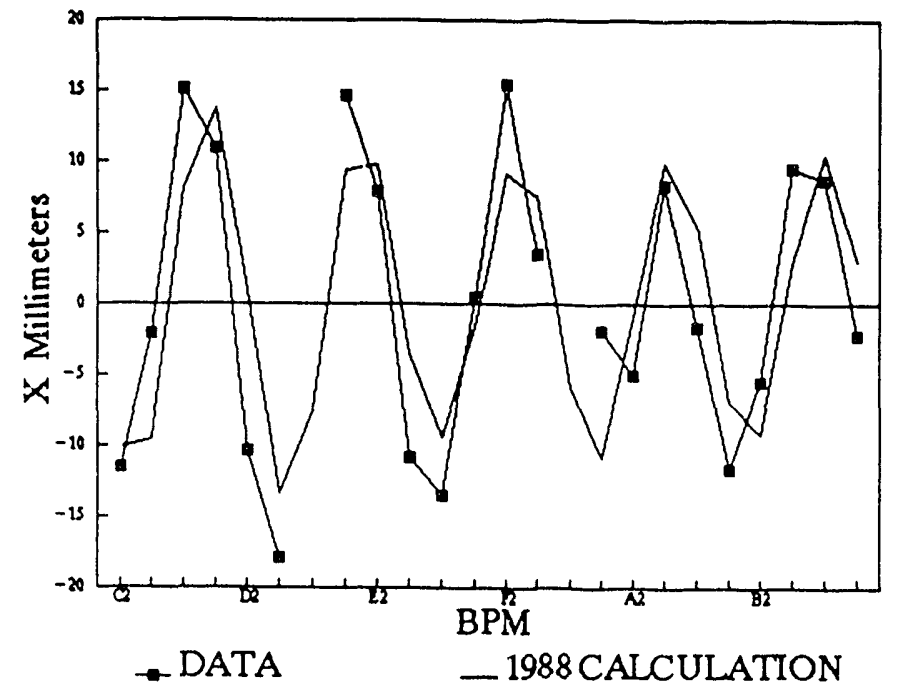

Figure 5.

The measured data of Figure 1 plotted along with a curve generated in the 1988 design study which assumed a random distribution of survey errors. The conclusion is that the measured orbit could well result from a random distribution of survey errors, which were within our rms tolerances of 0.3 millimeters.

\section{REFERENCES}

[1] W.T. Weng, "Operation of the Brookhaven AGS with the Booster", these proceedings.

[2] D.J. Ciardullo et.al, "Design and Performance of the Booster Beam Position Monitor System", Proc. XVth Intern. Conf. High Energy Accelerators, Hamburg, Germany, July 20-24, 1992, Ed. J. Rossbach, Int. J. Mod. Phys. A, 2A, Vol. I, 245-247 (1993).

[3] E. Wilson, "Transverse Beam Dynamics", p.78; CERN Accelerator School, CERN85-19, Geneva, 1985

[4] G. Danby and J.W. Jackson, "Vacuum Chamber Eddy Current Self-Correction for the AGS Booster Accelerator", Proc. XIV Intem. Conf. High Energy Accelerators, Tsukuba, Japan, August 22-26, 1989, Ed D. Keefe, Particle Accelerators 27, Part II, 33-38 (1990). 

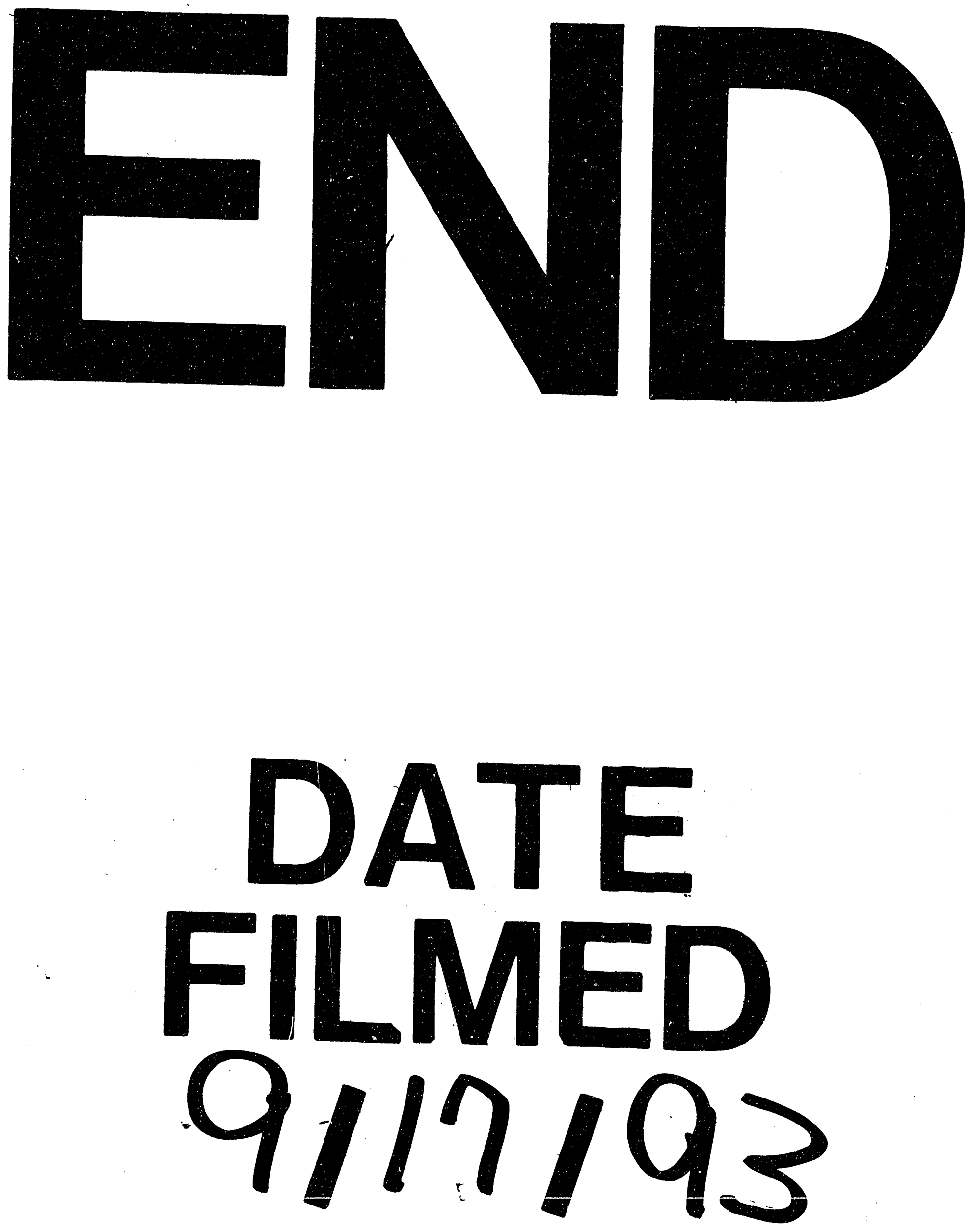
